\title{
A counter-intuitive method for slowing down an analyte in a nanopore and its application to polymer sequencing and analysis
}

\author{
G. Sampath
}

\begin{abstract}
A major obstacle in nanopore sensing for polymer sequencing, identification, and chemical analysis is the high translocation speed of an analyte (nucleotide, DNA, amino acid (AA), peptide) through the pore, about 10-100 monomers/ $\mu \mathrm{s}$, which exceeds the capability of currently available detectors. Attempts to resolve the problem fully have been less than satisfactory. Here a counter-intuitive method based on reversing the pore voltage and/or increasing analyte mobility by changing the solution $\mathrm{pH}$ is described. A simplified FokkerPlanck (F-P) model shows translocation times of 1-10 ms. More realistic simulations show that a bi-level positive-negative voltage profile can trap an analyte inside the pore for $\sim 1 \mathrm{~ms}$, which can be detected with a bandwidth of 1-10 Khz. This is a comprehensive approach to slowdown that can be used for free nucleotides, single amino acids, oligonucleotides/DNA strands, and oligopeptides/proteins. The applicability of this method to existing nanopore analysis techniques and issues relating to implementation are discussed.
\end{abstract}

\section{Introduction}

In recent years nanopores [1,2] have emerged as a single-molecule alternative to established methods of polymer sequencing and analysis. Most reports of nanopore studies of an analyte (which could be a DNA/RNA strand, oligonucleotide, protein/peptide, free nucleotide, free amino acid, or, sometimes, a normally uncharged polymer such as polyethylene glycol (PEG)) are usually focused on one or more of four analytical functions: detection, identification, sequencing, and chemical analysis (including chemical interactions, structure, posttranslational and other modifications). A fifth function, guaranteed delivery of the analyte to the entrance of the nanopore, is addressed in the discussion segment near the end of this communication. Nanopores are on the way to becoming a viable alternative for DNA sequencing at the single molecule level [3]. Nanopore protein sequencing is yet to show a similar degree of success [4].

Nanopore analysis is based on the use of an electrolytic cell (e-cell) in which a polymer translocates under the influence of an electrical potential through a nano-sized pore in a thin membrane separating two chambers known as cis and trans that contain an electrolyte (such as $\mathrm{KCl}$ ). One or the other of the following is used to perform one of the four functions mentioned above: 1) the size of the resulting current blockade [1-3]; 2) optical detection of fluorophore labels attached to the analyte [5]; and 3) transverse current measurements lateral to the pore [6].

A major problem in nanopore sensing is the high speed with which an analyte translocates through the pore, typically $10 \mathrm{~s}$ to $100 \mathrm{~s}$ of monomers per $\mu \mathrm{s}$. A number of solutions have been proposed and studied. With one exception (discussed below), the best slowdown achieved thus far is by about two orders of magnitude. Most methods are designed for a particular analyte, usually single DNA strands. A more general solution that can be applied to a range of analyte molecules is yet to be reported.

The present communication attempts to end this impasse by introducing a method that can be applied to a variety of analytes, including nucleotides, AAs, oligonucleotides, and peptides. A counter-intuitive approach to slowing down that is based on reversing the pore voltage and/or increasing the analyte's mobiliy is introduced and a Fokker-Planck model of translocation presented. A more realistic model based on a tandem pore structure with a positive-negative voltage profile is then described. Simulation results are presented to show that translocation times of $\sim 1 \mathrm{~ms}$ in a tandem nanopore of length $11 \mathrm{~nm}$ can be achieved for the four types of analytes mentioned above. The paper concludes with a discussion of the applicability of the method to existing methods of nanopore sensing and also looks at implementation issues.

\section{Nanopores for polymer analysis: Sequencing, detection, identification, and structure}

1) Nanopore studies of DNA. Much of the impetus for nanopore research has arisen in the context of DNA sequencing. There are broadly two approaches: 'strand sequencing' and 'exonuclease sequencing'. In 'strand sequencing' an intact strand of DNA passes through the pore under the influence of an electric potential across the pore. Changes that occur in the quiescent current are measured and related to the bases in the strand by deconvolution of the pore current signal. To reduce the signal processing bandwidth required a procedure known as 'sequencing by synthesis' (SBS) is used [7,8], in which a single DNA strand is ratcheted through the pore by an enzyme motor while the complementary strand is synthesized. SBS is prone to base calling errors because of the likelihood of the strand reversing direction. A method of decreasing such errors is described in [9]. In 'exonuclease sequencing' [10], an exonuclease enzyme covalently bonded to the cis side of a biological pore (such as alpha-hemolysin or AHL) is used to successively cleave the leading nucleotides in the strand; the latter fall through the pore. The resulting blockades are separated in time and space so that, ideally, no signal deconvolution is necessary. However, a cleaved base may diffuse back into the cis bulk and be missed or be 
called out of order. To remedy this an e-cell with two pores in tandem with the exonuclease enzyme covalently bonded to the downstream side of the first pore has been proposed [11]. A Fokker-Planck model was used to show that cleaved bases can be called in natural order. Furthermore simulations in [12] have shown that with appropriate pore and cis chamber sizes, the loss to diffusion is close to zero.

Blockade-based detection requires a considerable degree of measurement precision to be able to distinguish among the blockades due to the four nucleobases (A, T, C, and G). Optical methods based on fluorescent labels [13] require much less precision. Alternatively transverse electronic measurements of the current across the pore can be measured with 1 to 2 orders of magnitude higher than longitudinal blockade currents [14].

Identification of DNA sequences is a simpler problem than sequencing. Plasmonic methods [15], which are based on measuring changes in the refractive index of light due to an analyte, can be used for this. Nanopores have also been used for structural analysis of DNA [16] and in studying post-translational modifications (PTMs) such as methylation of single bases [17].

2) Nanopore studies of proteins. Nanopore protein sequencing is more difficult than DNA sequencing because there are 20 amino acids to identify, as opposed to 4 bases in DNA. A variety of methods are known, some are theoretical [18,19] while others are practical studies [20,21] that are yet to be developed fully. In [18] the tandem pore structure in [11] is used with an exopeptidase replacing the exonuclease. Protein sequencing with a pair of orthogonal channels is proposed in [19]. In [20] transverse electrodes at the pore junction have small recognizer molecules attached to the pore that match with specific amino acids. In [21] single free AAs or residues within a peptide are identified from the blockade current. For recent reviews of protein sequencing/sensing with nanopores, see $[22,23]$.

As with DNA, protein identification is simpler than sequencing as it suffices to find a partial sequence and look for a matching subsequence in a proteome sequence database, see, for example, [24] and [25]. Detection of a protein may be even simpler, without requiring database lookup, for example through matching carrier molecules [26]. In [27] the use of an electro-osmotic trap to study conformation changes in a folded protein is described. In [28] folding and mis-folding of a protein are examined with a nanopore. PTMs in proteins have also been studied with nanopores, an example is phosporylation of a protein [29].

Translocation speeds in a nanopore and methods to slow down an analyte

In all of the applications considered above, the nanopore approach, faces a major difficulty. The analyte translocates through the pore at a high rate (10-100 monomers per microsecond), which is more than the detector's bandwidth can handle. This is a 20 -year-old problem for which a wide-ranging solution has remained elusive; most efforts are limited to one type of analyte, usually DNA. Two reviews separated by a decade show that progress is still slow [30,31]. Methods include: magnetic or optical tweezers [32,33], increasing the viscosity of the solution in the e-cell [34], use of an RTIL (room temperature ionic liquid) for the electrolyte [35], binding the protein with SDS (Sodium Dodecyl Sulfate) to allow time for unfolding before the protein enters the pore (and also invest the protein with a higher and uniform electrical charge) [36], threading an analyte string through dual side-by-side nanopores to control its motion [37], and a ring of field effect transistor (FET) gates to control the rate of entry of the analyte into the pore [38]. The use of an enzyme motor to ratchet a strand of DNA through the pore [7,8] has already been noted above; this by far is the most effective method known. However it only applies to DNA strands.

\section{A counter-intuitive approach to analyte slowdown}

A method for slowing down a general analyte in a nanopore that runs counter to many of the approaches mentioned above is introduced next. It is based on reversing the voltage across the pore and increasing the mobility of some analytes. A simplified Fokker-Planck model of analyte translocation in the method presented here shows significant increases in translocation times, up from tens of nanoseconds to the millisecond range, for the four nucleotides (dAMP, dTMP, dCMP, dGMP), the 20 amino acids (AAs), short homonucleotides and heteronucleotides, and short homopeptides and heteropeptides. Simulations that are more realistic than the model show that a bi-level positive-negative voltage profile across the nanopore can yield translocation times of $\sim 1 \mathrm{~ms}$, which can be detected with a bandwidth of 1-10 Khz. 

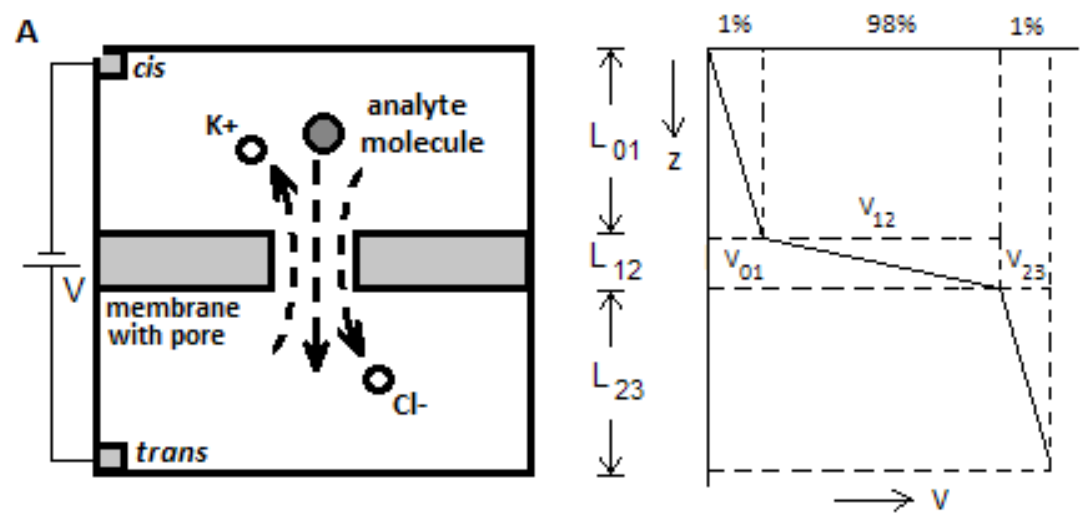

B

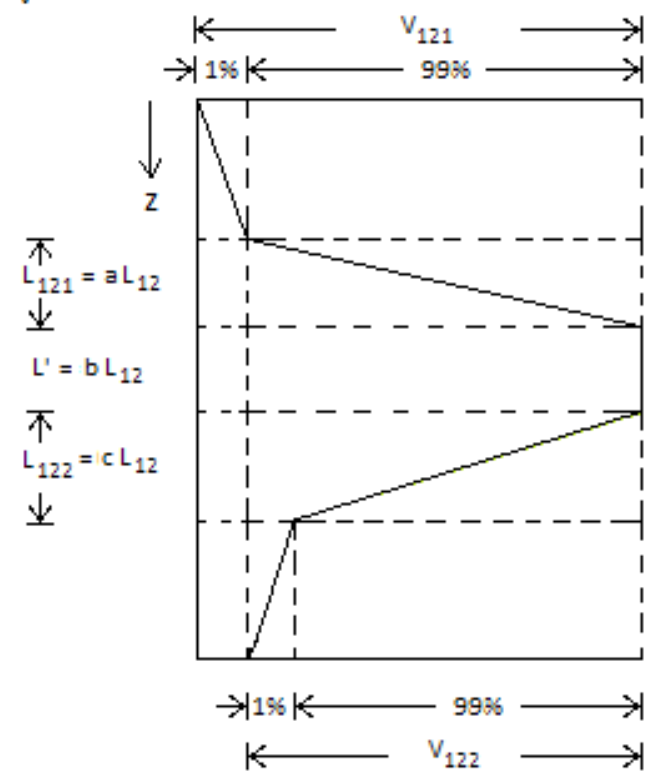

Figure 1. Schematic of electrolytic cell (e-cell); membrane containing nanopore separates cis and trans chambers containing salt $(\mathrm{KCl})$ solution. (A) Ionic current flow in e-cell is due to $\mathrm{K}^{+}$and $\mathrm{Cl}^{-}$ions; analyte translocating through pore causes reduction of open pore current; (B) $98 \%$ of voltage $\mathrm{V}$ applied between cis and trans drops across pore; (C) Two pores in tandem with different voltages across each, with intervening conducting membrane layer; (D) Positive voltage $V_{121}$ across upstream pore of length $L_{121}=a L_{12}$, with $99 \%$ dropping across pore, the other $1 \%$ across cis; conducting membrane layer at constant potential over thickness $\mathrm{bL}_{12}$; negative voltage $\mathrm{V}_{122}$ across downstream pore of length $\mathrm{L}_{122}=\mathrm{cL}_{12}$, with $99 \%$ dropping across pore, the other $1 \%$ across trans. In simulations $\mathrm{L}_{12}=11 \mathrm{~nm}, \mathrm{a}=\mathrm{c}=5 / 11, \mathrm{~b}=1 / 11$. Voltages $\mathrm{V}_{121}$ and $\mathrm{V}_{122}$ are varied to give different profiles so that analyte can be trapped for long durations; their values depend on analyte and $\mathrm{pH}$ of solution in e-cell, see Figure 2.

Consider an e-cell with cis and trans chambers filled with an electrolyte $(\mathrm{KCl})$ and separated by a thin membrane containing a nano-sized pore (Figure 1A). A voltage V (cis negative to trans positive) ionizes the electrolyte; $\mathrm{Cl}$ - ions flow from cis to trans through the pore, $\mathrm{K}+$ ions from trans to cis, The direction of flow of an analyte normally depends the electrical charge it carries, while the translocation speed is determined by its mobility, which is proportional to the charge. dAMP, dTMP, dCMP, and dGMP carry roughly the same amount of negative charge and go from cis to trans. The 20 amino acids in $\mathbf{A A}=$ $\{A, N, Q, G, I, L, M, F, P, S, T, W, V, R, K, D, E, C, H, Y\}$ carry a charge equal to $C_{\text {mult }} q . C_{\text {mult }}$ is a multiplier between 0 and \pm 2 and depends on the $\mathrm{pH}$ level of the solution, $\mathrm{q}$ is the electron charge. $\mathrm{C}_{\text {mult }}$ can be calculated with the Henderson-Hasselbalch (H-H) equation [39]; see Supplement for analyte mobility tables. To reduce the number of AAs to consider when devising methods to slow them down, they are divided into equivalence classes. Two such are:

$$
\begin{aligned}
& \mathbf{A A}=\{\{\mathrm{A}, \mathrm{N}, \mathrm{Q}, \mathrm{G}, \mathrm{I}, \mathrm{L}, \mathrm{M}, \mathrm{F}, \mathrm{P}, \mathrm{S}, \mathrm{T}, \mathrm{W}, \mathrm{V}\},\{\mathrm{R}, \mathrm{K}\},\{\mathrm{D}, \mathrm{E}\},\{\mathrm{C}\},\{\mathrm{H}\},\{\mathrm{Y}\}\} \\
& \mathbf{A A}=\{\{\mathrm{A}, \mathrm{N}, \mathrm{Q}, \mathrm{G}, \mathrm{I}, \mathrm{L}, \mathrm{M}, \mathrm{F}, \mathrm{P}, \mathrm{S}, \mathrm{T}, \mathrm{W}, \mathrm{V}\},\{\mathrm{R}, \mathrm{K}, \mathrm{D}, \mathrm{E}, \mathrm{C}, \mathrm{H}, \mathrm{Y}\}\} .
\end{aligned}
$$

Two AAs are in the same class if their maximum absolute mobilities are roughly the same. 
To calculate the mobilities of oligonucleotides and oligopeptides, these polymers are modeled as rigid rods. The details are given in the Supplement.

In the absence of a transmembrane voltage all analytes diffuse through the solution, with the direction of transport determined by boundary conditions. In general the analyte causes a reduction or blockade in the normal pore current but this also depends on other factors such as the electro-osmotic potential due to counterions in the electrolyte and residual charges on the pore wall. Here a Fokker-Planck (F-P) equation is used to model analyte motion in the e-cell in Figure 1A and compute the dwell/translocation time of an analyte in/through the pore. The mathematical formulation is given in the Supplement; only the essential features are presented here.

A 1-dimensional approximation is applied to the nanopore in Figure 1A over $0 \leq \mathrm{z} \leq \mathrm{L}_{12}$ for time $\mathrm{t} \geq 0$. Let $\mathrm{T}$ be the time of translocation of an analyte through the pore and $\mathrm{E}(\mathrm{T})$ its mean. Let $\mathrm{D}$ be the diffusion constant, $\mu$ the analyte mobility, and $\mathrm{v}_{\mathrm{z}}$ the drift velocity through the pore $=\mu \mathrm{V}_{12} / \mathrm{L}$. With $\alpha=\mathrm{v}_{\mathrm{z}} \mathrm{L} / \mathrm{D}=\mu \mathrm{V}_{12} / \mathrm{D}, \mathrm{V}_{12}=\mathrm{V}_{\text {, and } \mathrm{L}_{12}}$ $=\mathrm{L}$, it can be shown that

$$
E(T)=\left(L^{2} / D \alpha\right)\left[1-(1 / \alpha)\left(1-\mathrm{e}^{-\alpha}\right)\right]
$$

The following three cases can be considered:

$$
\begin{array}{lll}
\alpha>0: & \mathrm{E}(\mathrm{T}) \approx \mathrm{L}^{2} / \mu \mathrm{V} & \\
\alpha=0: & \mathrm{E}(\mathrm{T}) \approx \mathrm{L}^{2} / 2 \mathrm{D} & \text { (pure diffusion) } \\
\alpha<0: & \mathrm{E}(\mathrm{T}) \approx\left(\mathrm{L}^{2} \mathrm{D} / \mu^{2} \mathrm{~V}^{2}\right) \mathrm{e}^{|\mu \mathrm{V} / \mathrm{D}|} &
\end{array}
$$

Equation $3 \mathrm{c}$ shows that reversing the voltage $V_{12}$ across the pore and increasing the mobility of an analyte (by changing the $\mathrm{pH}$ of the solution) can significantly increase the translocation time. With the voltage reversed and the mobility raised, the numerator in Equation $3 \mathrm{c}$ has the term $\mathrm{e}^{|\mu \mathrm{V} / \mathrm{D}|}$ while the denominator has the term $\mu^{2} \mathrm{~V}^{2}$. The numerator rises much more rapidly than the denominator to cause a large increase in the translocation time (Table 1). This is of course due to the front end wall of the pore being considered a reflector in the simplified F$\mathrm{P}$ model. Table 1 shows translocation times in a nanopore of length $10 \mathrm{~nm}$ for dAMP, five of the 20 AAs, one homonucleotide made up of 10 repeating dAMP molecules, one random heteronucleotide of length 10, one homopeptide made up of 10 repeating R (arginine) molecules, and three random heteropeptides of length 10 . In the last case the first has both charged and uncharged residues, the second has one charged residue, and the third is the same as the second except that the charged residue is replaced with an uncharged residue. This mix is chosen to cover all possible cases of interest.

Table 1 Mean translocation times $\mathrm{E}(\mathrm{T})$ for $\mathrm{dAMP}$, five AAs, a homopolynucleotide, a random heteropolynucleotide, a homopolypeptide, and three random heteropolypeptides calculated with Equation $3 \mathrm{c}$ in the tandem pore of Figure $1 \mathrm{~A}$ with $\mathrm{L}_{12}=10 \mathrm{~nm}$. $\mathrm{pH}$ value and voltage required to raise times to millisecond range are shaded. $A n=$ analyte; $D=$ diffusion constant; $\mu=$ mobility; $V_{12}=$ pore voltage $\left(=V_{12}\right.$ in Figure 1$)$.

\begin{tabular}{|c|c|c|c|c|c|c|c|}
\hline $\mathbf{A n}$ & $\mathbf{p H}$ & $\mathbf{D}\left(\mathbf{1 0}^{-\mathbf{1 0}} \mathbf{m}^{2} \mathbf{s}\right)$ & $\boldsymbol{\mu}\left(\mathbf{1 0}^{-\mathbf{8}} \mathbf{m}^{2} / \mathbf{V s}\right)$ & $\mathbf{V}(\mathbf{v o l t})$ & $\mathbf{E}(\mathbf{T})(\mathbf{m s})$ & $\mathbf{V}(\mathbf{v o l t})$ & $\mathbf{E}(\mathbf{T})(\mathbf{m s})$ \\
\hline dAMP & 7 & 4.47 & -1.74 & -0.4 & 6.49 & 0.4 & $1.63 \times 10^{-5}$ \\
\hline Ala & 11 & 8.21 & 3.19 & -0.4 & $8.86 \times 10^{-6}$ & 0.4 & 3.51 \\
\hline Arg & 7 & 6.06 & -2.36 & 0.4 & 4.65 & -0.4 & $1.20 \times 10^{-5}$ \\
\hline Cys & 9 & 6.76 & 2.95 & 0.4 & $9.66 \times 10^{-6}$ & -0.4 & 22.8 \\
\hline His & 11 & 6.25 & 2.32 & 0.4 & $1.22 \times 10^{-5}$ & -0.4 & 2.46 \\
\hline Tyr & 10 & 6.11 & 2.69 & 0.4 & $6.64 \times 10^{-5}$ & -0.4 & 28.5 \\
\hline AAAAAAAAAA & 7 & 2.62 & -99.42 & -0.005 & 1.26 & 0.1 & $1.22 \times 10^{-6}$ \\
\hline CCAGTTTATA & 7 & 2.63 & -99.42 & -0.005 & 1.21 & 0.1 & $1.12 \times 10^{-6}$ \\
\hline RRRRRRRRRR & 7 & 3.55 & -13.8 & -0.055 & 1.48 & 0.1 & $8.54 \times 10^{-6}$ \\
\hline HWVEDVDLTP & 12 & 4.00 & -6.22 & -0.1 & 7.13 & 0.2 & $9.41 \times 10^{-6}$ \\
\hline IVHSMSWALP & 12 & 4.13 & -1.60 & -0.4 & 6.56 & 0.1 & $5.64 \times 10^{-5}$ \\
\hline IVFSMSWALP & 12 & 4.15 & -1.61 & -0.4 & 6.53 & 0.1 & $5.62 \times 10^{-5}$ \\
\hline
\end{tabular}


In what follows, the analyte is assumed to be negatively charged. The development can be extended to positively charged analytes with a systematic change of sign.

The F-P model above considers the pore in isolation and assumes that it is reflective on the inside. The pore, however, is part of a 3-stage continuum (cis, pore, trans), with the analyte added at the top of the cis chamber. With V set to the normal polarity (cis negative, trans positive), the analyte diffuses through cis to the pore entrance and then translocates through the pore to trans under the influence of diffusion and electrophoresis. The mean translocation time is a few (10-100) nanoseconds, which is beyond the capability of the detector. With the tandem pore structure of Figure 1C, no such reflective boundary exists for the upstream pore, which is the first stage of the structure [upstream pore - conducting layer - downstream pore] and is open at both ends. If $\mathrm{V}$ is reversed, then for the F-P model to be applicable the analyte must translocate from cis to the pore entrance without being lost to diffusion and enter the pore; and the pore entrance must behave, at least approximately, like a reflector when the analyte is inside the pore. This can be achieved with a tandem pore of the form pore segment $[0, a L]$ : conducting layer $[a L, a L+b L]$ : pore segment $[a L+b L, a L+b L+c L] ; \mathrm{a}+\mathrm{b}+\mathrm{c}=1$, and a bi-level voltage profile. The profile has normal polarity over $[0, a \mathrm{~L})$, is zero over $[\mathrm{aL}, \mathrm{aL}+\mathrm{bL}]$, and has the opposite polarity over $[a L+b L, a L+b L+c L]$. Figure $1 \mathrm{D}$ shows such a profile with $\mathrm{a}=\mathrm{c}=5 / 11$ and $\mathrm{b}=1 / 11$; the voltage profile is written as (positive voltage segment, voltage over conducting layer $(=0)$, negative voltage segment), for example $(0.35,0,-0.5)$.

Now consider the forces on an analyte in this tandem structure. With a positive voltage applied to the upstream pore the analyte is forced toward the downstream pore: the upstream pore with the positive voltage is acting like a reflecting boundary for the downstream pore with the negative voltage. One can think of it as a soft or spongy reflector. Increasing the positive voltage increases this 'reflective' effect and causes the analyte to move further toward the upstream pore, where it encounters an opposing field that effectively slows down its progress. The values of the positive and negative voltages can be set appropriately to obtain slowdown of an analyte. The right balance will depend on the analyte and the solution $\mathrm{pH}$ and can be obtained approximately by simulation (as has been done here) and more accurately by experiment.

A rough estimate of the expected translocation time $\mathrm{E}_{12}(\mathrm{~T})$ can be obtained for the tandem pore structure in Figure $1 \mathrm{C}$ by separately calculating the times for the three segments of the pore (by using Equations $3 \mathrm{a}, 3 \mathrm{~b}$, and $3 c)$ and adding them. However because of diffusion the above estimate can be way off as the analyte goes through four discontinuities: (cis : pore segment with positive voltage); (pore segment with positive voltage : conducting layer); (conducting layer : pore segment with negative voltage); and (pore segment with negative voltage : trans). One way to get better estimates is to formulate a discontinuous F-P equation for the five-stage structure in Figure 1C and solve it numerically. Here an alternative approach based on simulation is taken.

\section{Simulating slowdown with a tandem nanopore}

The slowdown process described above was simulated by assuming an analyte molecule to be a dimensionless particle. The procedure is similar to that in [12]; the details are available in the Supplement. The nine analytes in Table 1 were used in the simulations. Thus: dAMP represents all five nucleotides; Arginine represents the AA subset $\{\mathrm{R}, \mathrm{K}, \mathrm{D}, \mathrm{E}, \mathrm{C}, \mathrm{H}, \mathrm{Y}\}$ (Equation 1a); Alanine represents $\{\mathrm{A}, \mathrm{N}, \mathrm{Q}, \mathrm{G}, \mathrm{I}, \mathrm{L}, \mathrm{M}, \mathrm{F}, \mathrm{P}, \mathrm{S}, \mathrm{T}, \mathrm{W}, \mathrm{V}\}$ (Equation 1b) at two different values of $\mathrm{pH}$; AAAAAAAAAA is a sample homonucleotide; CCAGTTTATA is a randomly generated heteronucleotide; RRRRRRRRRR is a homopeptide ; HWVEDVDLTP is a random heteropeptide with a mix of charged and uncharged residues; IVHSMSWALP is a random heteropeptide with one charged residue (H); and IVFSMSWALP is almost the same as the previous analyte except that the uncharged residue F replaces the charged residue $\mathrm{H}$. This set covers all possible test cases of interest.

Under the influence of the bi-level voltage profile an analyte may oscillate between cis and the pore, stay for extended times inside the pore, or translocate fully to exit into trans. In the absence of full translocation the longest dwell time of the analyte during the simulation run is used. Thus an analyte could remain trapped inside the pore for sufficiently long times that the detector can detect it based on a pore current blockade or by optical means. As noted earlier, to get millisecond-level translocation times it is necessary to increase the positive voltage segment of the profile. Figure 2 below shows the increases in translocation times achieved with different bi-level voltage profiles for the above listed analytes. Notice how the time for Alanine jumps by $\sim 4$ orders of magnitude with the third voltage profile when the $\mathrm{pH}$ goes from 7 to 11 in Figure 2A. Notice also the very small negative voltage for AAAAAAAAAA, CCAGTTTATA, and RRRRRRRRRR, which is sufficient to get a trapping time of $\sim 1 \mathrm{~ms}$ (Figure $2 \mathrm{~B}$ ). With more negative voltages the analyte can be trapped for an indefinite length of time; this was observed in the simulations but the results are not included in the data presented here. 
A

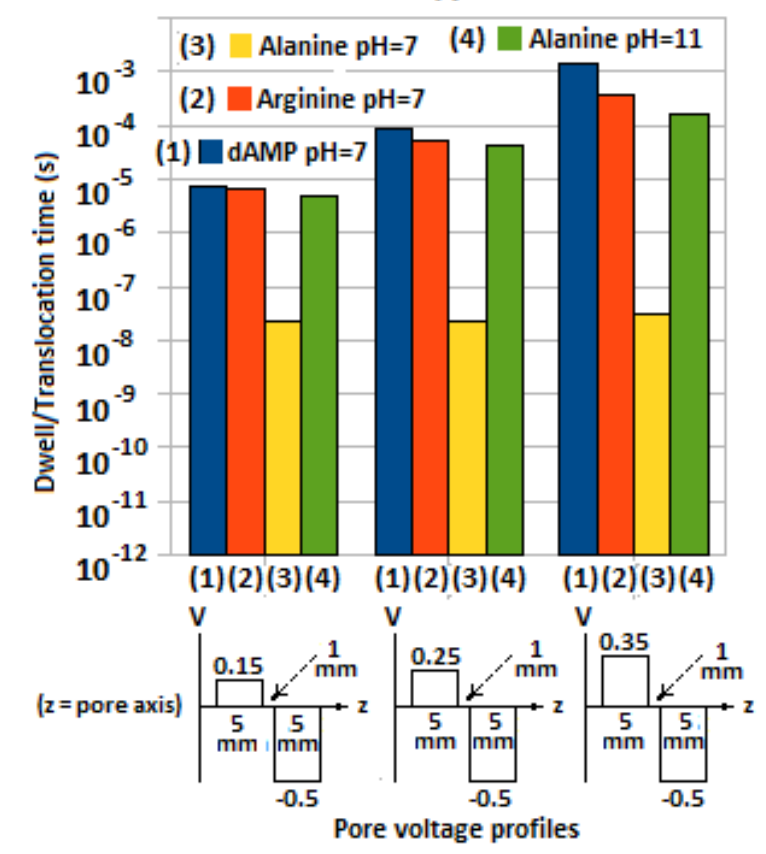

B

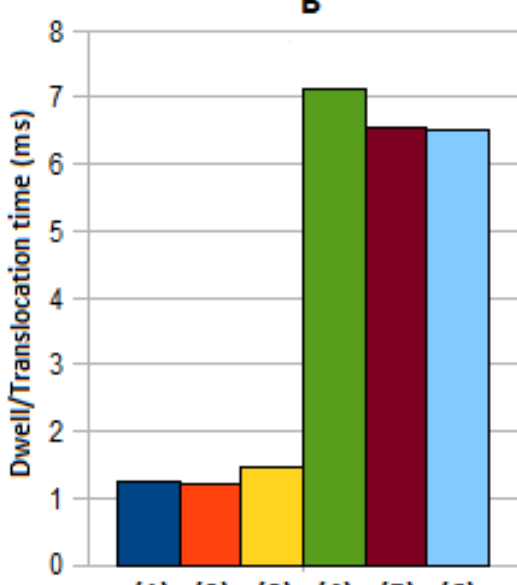

(1) (2) (3) (4) (5) (6)

(1) $\square$ AAAAAAAAAA

(2) $\square$ CCAGTTTATA

(3) $\square$ RRRRRRRRRR

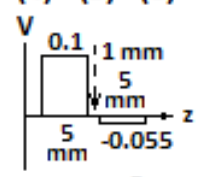

Pore voltage profiles
口HWVEDVDLTP (4)

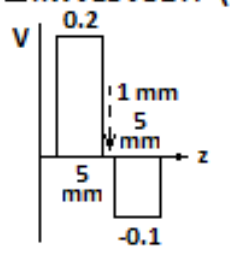

IIVHSM SWALP (5)

DIVFSMSWALP (6)

$\mathrm{v}$

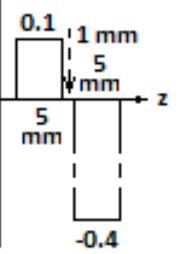

Figure 2. Mean translocation times of nine analytes for different voltage profiles over tandem pore structure in Figure 1C. Length specs on z-axis correspond to upstream pore thickness, conducting layer thickness, and downstream pore thickness (respectively $\mathrm{L}_{121}=\mathrm{aL}_{12}, \mathrm{~L}^{\prime}=\mathrm{bL}_{12}$, and $\mathrm{L}_{122}=\mathrm{cL}_{12}$ in Figure 1); values $\mathrm{a}=\mathrm{c}=5 / 11, \mathrm{~b}$ $=1 / 11$, and $\mathrm{L}_{12}=\mathrm{L}=11 \mathrm{~nm}$ used in simulations. Voltage levels in each profile correspond to the triple (positive voltage across upstream pore, conducting layer potential, negative voltage across downstream pore), used in simulations. Voltage axis in volts; time data correspond to larger of dwell time and translocation time through tandem pore structure. (A) Translocation times for dAMP, Arg, and Ala with different voltage profiles and $\mathrm{pH}$ values; (B) Translocation times for two oligonucleotides and four oligopeptides with different voltage profiles (pH values vary, not shown). (Histogram bars numbered to aid monochrome viewing.)

Effect of bi-level voltage profile on ionic current. An unintended consequence of the bi-level voltage profile is that it also decreases the pore ionic current $\mathrm{I}_{\text {pore. }}$. This decrease can be estimated from the translocation/dwell times of ions in the pore. Table 2 below shows the current level due to $\mathrm{Cl}$ - ions flowing from cis to trans, which results in a net decrease in the current. As the normal $\mathrm{K}+$ ion flow from cis to trans is small the effect of the voltage profile on it is much smaller. A decrease similar to that with $\mathrm{Cl}$ - going from cis to trans occurs in the $\mathrm{K}+$ current from trans to cis. Since the current delivered by an ion from cis to trans is inversely proportional to the translocation time through the pore that separates the two chambers the result is a steep fall in the current to $\sim 10 \%$ of that without the opposing voltage. If the negative segment voltage is low (for example, see profiles in Figure 2B for AAAAAAAAAA, CCAGTTTATA, and RRRRRRRRRR) this reduction is negligible. However, even with a peptide that carries a low charge, regardless of whether it is a homopeptide or not, it can be invested with a high charge by attaching a short homopeptide made up from a charged residue. For example RRRRR can be attached to the uncharged heteropeptide IVFSMWALP to get RRRRRIVFSMWALP. This can be slowed down with a voltage of only -0.05 just like the other homonucleotides and homopeptide in Table 1 so that the reduction in ionic current because of the negative voltage segment is marginal.

Table 2. Shows increased translocation times of ions (and therefore lower base currents) in a tandem pore with positive-negative voltage profile of the form (positive voltage segment, conductor layer, negative voltage segment). Total pore length $=11 \mathrm{~nm}$. With low negative voltages, effect is negligible, not shown (see text above).

\begin{tabular}{|c|c|c|c|c|c|}
\hline \multirow{2}{*}{$\begin{array}{c}\text { Voltage } \\
\text { profile } \\
(\mathrm{V}, \mathrm{V}, \mathrm{V})\end{array}$} & \multicolumn{2}{|c|}{$\begin{array}{l}\text { Translocation } \\
\text { time with } \\
\text { voltage profile } \\
\left(10^{-8} \mathrm{~s}\right)\end{array}$} & \multirow[t]{2}{*}{$\begin{array}{c}\text { Normal } \\
\text { pore } \\
\text { voltage }\end{array}$} & \multicolumn{2}{|c|}{$\begin{array}{l}\text { Translocation } \\
\text { time with normal } \\
\text { pore voltage }\left(10^{-8}\right. \\
\text { s) }\end{array}$} \\
\hline & Cl- & $\mathrm{K}+$ & & $\mathrm{Cl}-$ & $\mathrm{K}+$ \\
\hline
\end{tabular}




\begin{tabular}{|c|c|c|c|c|c|}
$(0.5,0,-0.12)$ & 5.83 & 5.98 & 0.15 & 0.55 & 0.57 \\
\hline$(0.5,0,-0.15)$ & 12.4 & 12.6 & 0.25 & 0.85 & 0.88 \\
\hline$(0.5,0,-0.1)$ & 3.69 & 3.79 & 0.35 & 0.41 & 0.420 \\
\hline
\end{tabular}

\section{Discussion}

The following are some pertinent observations on the slowdown method introduced above.

1) The method is fairly straightforward as it does not require any special devices or chemicals. The only extra requirement is constructing two pores in tandem with a conducting layer in between for use as a terminal between the two voltage levels (Figure 1C). This can be done more easily with solid-state pores than with biological pores. Furthermore, voltages can be higher with the former, whereas with a biological pore they are limited by electrical breakdown to about $300 \mathrm{mV}$, which in many cases may be too low to allow significant slowdowns. This is also borne out by the simulation results in Table 1, which shows that for some analytes -400 $\mathrm{mV}$ or more may be required in the negative voltage segment to get translocation times in the millisecond range. This should not be a problem with synthetic membranes. Thus with a silicon nitride membrane $5 \mathrm{~nm}$ thick, the pore voltage can be as high as $1 \mathrm{~V}[40]$.

2) The structure can be fabricated as a stack of three layers: two synthetic silicon-based membranes and a conducting membrane in between, graphene or aluminum may be used for the latter. Fabrication of nanoporebased structures is reviewed in [41]. Fabrication of a three-layer silicon-graphene-germanium structure is described in [42]. Alternatively $\mathrm{Si}++$ can be used for the conducting layer (similar to the use of grounded $\mathrm{Si}++$ substrates in semiconductor devices)..

3) The method given here is well suited for use with exonuclease sequencing. The drastic increase in the translocation time for a nucleotide (Figure 2) can help reduce considerably the high percentage of nucleotides being missed because of excessive translocation speeds [10].

4) As noted above the reduction in the pore current due to a bi-level voltage profile across the tandem pore may pose a problem in blockade-based detection of some analytes. In those cases where the pore current is not of a sufficient strength, other methods may be appropriate. For example, in [43] a fluorophore-labeled molecule passes from the unlit (dark) cis side of an e-cell to the lighted trans side where it emits light and is detected. Interestingly, if the pore is replaced with a channel that is open on one side and the slowdown method given here is used, the passage of the analyte through the channel could be detected optically with a low bandwidth detector.

5) With AAs, since different $\mathrm{pH}$ values may be required to achieve slowdown, they may need to be probed in different e-cells (which is easier than recycling into the same cell after changing the $\mathrm{pH}$ ). A method to separate AAs from a sample by routing 20 copies of the AA to 20 different e-cells (one for each of the 20 different AAs) is described in [44].This is a binary measurement that effectively converts AA detection from a high precision analog process to a low precision digital one. If optical detection with fluorophore labels is used then a single color label can be used for all 20 AAs. (As a matter of fact any color can be used for any AA.)

6) With some analytes the slowdown method described above is based on increasing the analyte's mobility, which depends on increasing the charge associated with the analyte by adjusting the $\mathrm{pH}$ of the solution. However the attraction of counterions to the analyte may reduce the charge carried by the analyte, and hence the mobility, by up to $25 \%$ [2].

7) Analyte translocation is a stochastic phenomenon so there is no guarantee that an analyte will enter the pore within a reasonable amount of time. One recent solution [45] uses a nanopipette to trap short DNA strands for transfer to a sensor like a nanopore. In the present work hydraulic pressure [46] has been used in all the simulations; see Figure S-2 in the Supplement. This results in an analyte introduced into the top of cis reaching the pore entrance without fail. There is, however, a tradeoff: the use of hydraulic pressure also prevents the flow of ions from trans to cis, thereby effectively halving the pore current.

8) An analyte may be trapped multiple times inside the pore. All sufficiently long dwells can then be recorded by the detector to increase the confidence level of the measurement. With $\mathrm{n}$ such dwells, multiple reads can be thought of as providing $\mathrm{nX}$ coverage. Incidentally, these reads can be done by repeatedly reversing the tandem pore voltage(s). (This will also require taking into account the rectification effect in nanopores, which arises from the forward and reverse conductances being different [47].)

\section{References}

[1] Z. Liu, Y. Wang, T. Deng, and Q. Chen, "Solid-State Nanopore-Based DNA Sequencing Technology", Journal of Nanomaterials 2016, 5284786. http://dx.doi.org/10.1155/2016/5284786

[2] M. Muthukumar, C. Plesa, and C. Dekker, "Single-molecule sensing with nanopores', Physics Today 68, 8, 40 (2015); doi: 10.1063/PT.3.2881 
[3] L. Reynaud, A. Bouchet-Spinelli, C. Raillon, and A. Buhot, "Sensing with nanopores and aptamers: a way forward", Sensors 20, 4495, 2020. doi:10.3390/s20164495

[4] N. Callahan, J. Tullman, Z. Kelman, and J. Marino. "Strategies for development of a next-generation protein sequencing platform". Trends Biochem. Sci. 2019. doi:10.1016/j.tibs.2019.09.005.

[5] T. Gilboa and A. Meller, "Optical sensing and analyte manipulation in solid-state nanopores", Analyst, 2015, $140,4733-4747$.

[6] M. Tsutsui, M. Taniguchi, K. Yokota, and T. Kawai, "Identifying single nucleotides by tunnelling current". Nat. Nanotechnol. 2010, 5, 286-90.

[7] K. R. Lieberman, G. M. Cherf, M. J. Doody, F. Olasagasti, Y. Kolodji, and M. Akeson, "Processive replication of single DNA molecules in a nanopore catalyzed by phi29 DNA polymerase," J. Am. Chem. Soc., 2010, 132, 17961-72.

[8] C. W. Fuller, S. Kumara, M. Porel, et al. "Real-time single-molecule electronic DNA sequencing by synthesis using polymer-tagged nucleotides on a nanopore array", PNAS 2016, 113, 5233-5238.

[9] M. T. Noakes, H. Brinkerhoff, A. H. Laszlo, I. M. Derrington, K. W. Langford, J. W. Mount, J. L. Bowman, K. S. Baker, K. M. Doering, B. I. Tickman, and J. H. Gundlach, "Increasing the accuracy of nanopore DNA sequencing using a time-varying cross membrane voltage", Nature Biotechnology, 37, 651-656 (2019).

[10] J. Clarke, H.-C. Wu, L. Jayasinghe, A. Patel, S. Reid, and H. Bayley, "Continuous base identification for single-molecule nanopore DNA sequencing", Nat. Nanotechnol. 2009, 4, 265-70.

[11] G. Sampath, "A tandem cell for nanopore-based DNA sequencing with exonuclease," RSC Adv., 5, 167-171, 2015.

[12] G. Sampath, "DNA sequencing with stacked nanopores and exonuclease: a simulation-based analysis", Electrophoresis 2016, 37, 2429-2434. doi: 10.1002/elps.201600049

[13] N. Klughammer and C. Dekker, "Palladium zero-mode waveguides for optical single molecule detection with nanopores", Nanotech. 2021. doi: 10.1088/1361-6528/abd976

[14] J. Prasongkit, A. Grigoriev, B. Pathak, R. Ahuja, and R. H. Scheicher, "Transverse conductance of DNA nucleotides in a graphene nanogap from first principles", Nano Lett. 2011, 11, 1941-5.

[15] A. Hubarevich, J.-A. Huang, G. Giovannini, A. Schirato, Y. Zhao, N. Maccaferri, F. De Angelis, A. Alabastri, and D. Garoli, "Lambda-DNA through a plasmonic nanopore - What can be detected by means of Surface Enhanced Raman Scattering?", arxiv.org 2020, arXiv:2004.00950 [physics.app-ph]. doi: 10.1021/acs.jpcc.0c06165

[16] K. Liu, P. Chao, K. Alexandre, P. N. Adrian, E. F. Georg, M. Olgica, and R. Aleksandra, "Detecting topological variations of DNA at single-molecule level", Nat. Communs. 2019, 10, 3.

[17] A. C. Rand, M. Jain, J. M. Eizenga, A. Musselman-Brown, H. E. Olsen, M. Akeson, B. Paten, "Mapping DNA methylation with high-throughput nanopore sequencing", Nat. Methods 2017, 14, 411-413.

[18] G. Sampath "Amino acid discrimination in a nanopore and the feasibility of sequencing peptides with a tandem cell and exopeptidase". RSC Adv. 5, 30694-30700, 2015.

[19] P. Boynton and M. Di Ventra, "Sequencing proteins with transverse ionic transport in nanochannels", Sci. Rep., 2016, 6, 25232.

[20] Y. Zhao, B. Ashcroft, P. Zhang, H. Liu, S. Sen, W. Song, J. Im, B. Gyarfas, S. Manna, S. Biswas, C. Borges, and S. Lindsay, "Single-molecule spectroscopy of amino acids and peptides by recognition tunneling", Nature Nanotechnol., 2014, 9, 466-473.

[21] H. Ouldali, K. Sarthak, T. Ensslen, F. Piguet, P. Manivet, J. Pelta, J. C. Behrends, A. Aksimentiev, and A. Oukhaled, "Electrical recognition of the twenty proteinogenic amino acids using an aerolysin nanopore", Nature Biotech. 2020, 38, 176-181. (Reviewed in: S. Howorka and Z. S. Siwy, "Reading amino acids in a nanopore", Nature Biotech. 2020, 38, 159-160. doi: 10.1038/s41587-019-0401-y)

[22] L. Restrepo-Pérez, C. Joo, and C. Dekker, "Paving the way to single-molecule protein sequencing", Nat. Nanotechnol. 2018, 13, 786-796. doi: 10.1038/s41565-018-0236-6

[23] M. Chinappi and F. Cecconi, "Protein sequencing via nanopore based devices: a nanofluidics perspective", J. Phys. Condens. Matter 2018, 30, 204002.

[24] M. Kolmogorov, E. Kennedy, Z. Dong, G. Timp, P. Pevzner, "Single-molecule protein identification by sub-nanopore sensors", PLOS Comp. Biol. 2017. doi 10.1371/journal.pcbi.1005356

[25] G. Sampath, "Protein fingerprinting with digital sequences of linear protein subsequence volumes: a computational study", J. Biosci. 2019, 44, 1-11.

[26] N. A. W. Bell, U. F. Keyser, "Specific protein detection using designed DNA carriers and nanopores", J. Am. Chem. Soc. 2015. doi 10.1021/ja512521w

[27] S. Schmid, P. Stömmer, H. Dietz, and C. Dekker, "Nanopore electro-osmotic trap for the label-free study of single proteins and their conformations", bioRxiv.org 2021. doi: https://doi.org/10.1101/2021.03.09.434634

[28] C. Madampage, O. Tavassoly, C. Christensen, M. Kumari, and J. S. Lee, "Nanopore analysis: an emerging technique for studying the folding and misfolding of proteins", Prion 2012, 6, 116-123. 
[29] C. B. Rosen, D. Rodriguez-Larrea, and H. Bayley, "Single-molecule site-specific detection of protein phosphorylation with a nanopore", Nature Biotechnol. 2014, 32, 179-181.

[30] U. F. Keyser, "Controlling molecular transport through nanopores", J. R. Soc. Interface 2011, 8, 1369-1378.

[31] Z. Yuan, Y. Liu, M. Dai, X. Yi, and C. Wang, "Controlling DNA translocation through solid-state nanopores", Nanoscale Res. Lett. 2020, 15. doi: 10.1186/s11671-020-03308-X

[32] U. F. Keyser, J. van der Does J, C. Dekker, and N. H. Dekker. "Optical tweezers for force measurements on DNA in nanopores". Rev. Sci. Instrum. 2006, 77, 105105. doi:10.1063/1.2358705.

[33] H. Peng and X. S. Ling. "Reverse DNA translocation through a solid-state nanopore by magnetic tweezers". Nanotech. 2009, 20, 185101. doi:10.1088/0957-4484/20/18/185101

[34] S. W. Kowalczyk, D. B. Wells, A. Aksimentiev, and C. Dekker. "Slowing down DNA translocation through a nanopore in lithium chloride". Nano Lett. 2012, 12, 1038-1044. doi:1 0.1021/n1204273h

[35] J. Feng, K. Liu, R. D. Bulushev, S. Khlybov, D. Dumcenco, A. Kis, and A. Radenovic. "Identification of single nucleotides in MoS2 nanopores". Nature Nanotech., 2015. doi: 10.1038/nnano.2015.219

[36] L. Restrepo-Pérez, S. John, A. Aksimentiev, C. Joo, and C. Dekker, "SDS-Assisted protein transport through solid-state nanopores", Nanoscale, 2017, 9, 11685. doi: 10.1039/c7nr02450a

[37] X. Liu, Y. Zhang, R. Nagel, W. Reisner, and W. B. Dunbar, "Controlling DNA tug-of-war in a dual nanopore device", arxiv.org, 27 Nov 2018. arXiv:1811.11105v1 [physics.bio-ph]

[38] M. Tsutsui, S. Ryuzaki, K. Yokota, Y. He, T. Washio, K. Tamada, and T. Kawai, "Field effect control of translocation dynamics in surround-gate nanopores", Nat. Communs. Mater. 2021, 2, 29. doi: 10.1038/s43246-021-00132-3

[39] D. L. Nelson and M. M. Cox, Lehninger's Principles of Biochemistry, 4th edn., W. H. Freeman, New York 2005 .

[40] A. Fragasso, S. Schmid, and C. Dekker, "Comparing current noise in biological and solid-state nanopores", ACS Nano 2020, 14, 1338-1349.

[41] Z. Yuan, C. Wang, X. Yi, Z. Ni, Y. Chen, and T. Li, "Solid-State Nanopore", Nanoscale Research Letters 2018, 13, 56. https://doi.org/10.1186/s11671-018-2463-Z

[42] C. Liu, W. Ma, M. Chen, W. Ren, and D. Sun, "A vertical silicon-graphene-germanium transistor", Nat. Communs. 2019, 10, 4873. doi: 10.1038/s41467-019-12814-1

[43] T. Auger, E. Bourhis, J. Donnez, A. Durnez, J. M. Di Meglio, L. Auvray, F. Montel, J. Yates, and J. Gierak, "Zero-mode waveguide detection of DNA translocation through FIB-organised arrays of engineered nanopores", Microelectron. Engg. 2018, 187-188, 90-94. https://doi.org/10.1016/j.mee.2017.12.005

[44] G. Sampath, "Label-free amino acid identification for de novo protein sequencing via tRNA charging and current blockade in a nanopore", bioRxiv, 2020.06.25.170803. doi:10.1101/2020.06.25.170803

[45] K. J. Freedman, L. M. Otto, A. P. Ivanov, A. Barik, S.-H. Oh, and J. B. Edel. "Nanopore sensing at ultralow concentrations using single-molecule dielectrophoretic trapping”, Nature Commun. 2016, 7, 10217. doi: $10.1038 /$ ncomms 10217

[46] H. Zhang, Q. Chen, Y. Wu, Y. Wang, X. Bei, and L. Xiao. "The temporal resolution and single-molecule manipulation of a solid-state nanopore by pressure and voltage", Nanotechnol. 2018, 29, 495501. doi: 10.1088/1361-6528/aae190

[47] T. Gamble, K. Decker,T. S. Plett, M. Pevarnik, J.-F. Pietschmann, I. Vlassiouk, A. Aksimentiev, and Z. S. Siwy, "Rectification of ion current in nanopores depends on the type of monovalent cations: Experiments and modeling", J. Phys. Chem. C 2014, 118, 9809-9819. doi: 10.1021/jp501492g

\section{Supporting information}

Supplementary information file with the following sections: Calculation of mobilities and diffusion coefficients; Table of mobility data; Formulation and analysis of Fokker-Planck model; Simulation details.

Email: sampath2068@yahoo.com 